\title{
Fast-food-based hyper-alimentation can induce rapid and profound elevation of serum alanine aminotransferase in healthy subjects
}

\author{
S Kechagias, ${ }^{1} \AA$ A Ernersson, ${ }^{1} \mathrm{O}$ Dahlqvist, ${ }^{2}$ P Lundberg, ${ }^{1,2}$ T Lindström, ${ }^{1} \mathrm{~F} \mathrm{H}$ Nystrom, ${ }^{1,3}$ \\ for the Fast Food Study Group
}

\begin{abstract}
See Commentary, p 568
${ }^{1}$ Division of Internal Medicine, Department of Medicine and Care, Faculty of Health Sciences, Linköping University, Linköping, Sweden; ${ }^{2}$ Division of Radiation Physics and Radiology, Center for Medical Image Science and Visualization, Department of Medicine and Care, Faculty of Health Sciences, Linköping University, Linköping, Sweden; ${ }^{3}$ Diabetes Research Centre. Linköping University, Linköping, Sweden
\end{abstract}

Correspondence to:

Dr Fredrik H Nystrom,

Department of Medicine and Care, Berzelius Science Park, University Hospital of Linköping, SE 58185 Linköping, Sweden; Fredrik.Nystrom@lio.se

Revised 25 October 2007 Accepted 28 October 2007 Published Online First 14 February 2008

\section{UNLOCKI}

This paper is freely available online under the BMJ Journals unlocked scheme, see http:// gut.bmj.com/info/unlocked.dtl

\section{ABSTRACT}

Objective: To study the effect of fast-food-based hyperalimentation on liver enzymes and hepatic triglyceride content (HTGC).

Design: Prospective interventional study with parallel control group.

Setting: University Hospital of Linköping, Sweden.

Participants: 12 healthy men and six healthy women with a mean (SD) age of 26 (6.6) years and a matched control group.

Intervention: Subjects in the intervention group aimed for a body weight increase of $5-15 \%$ by eating at least two fast-food-based meals a day with the goal to double the regular caloric intake in combination with adoption of a sedentary lifestyle for 4 weeks.

Main outcome measures: Weekly changes of serum aminotransferases and HTGC measured by proton nuclear magnetic resonance spectroscopy at baseline and after the intervention.

Results: Subjects in the intervention group increased from 67.6 (9.1) $\mathrm{kg}$ to 74.0 (11) $\mathrm{kg}$ in weight $(\mathrm{p}<0.001)$. Serum ALT increased from 22.1 (11.4) U/I at study start to an individual mean maximum level of 97 (103) U/I (range 19.4-447 U/I). Eleven of the 18 subjects persistently showed ALT above reference limits (women $>19 \mathrm{U} / \mathrm{l}$, men $>30 \mathrm{U} / \mathrm{ll}$ ) during the intervention. Sugar (mono- and disaccharides) intake during week 3 correlated with the maximal ALT/baseline ALT ratio $(r=0.62, p=0.006)$. HTGC increased from $1.1(1.9) \%$ to $2.8(4.8) \%$, although this was not related to the increase in ALT levels. ALT levels were unchanged in controls.

Conclusion: Hyper-alimentation per se can induce profound ALT elevations in less than 4 weeks. Our study clearly shows that in the evaluation of subjects with elevated ALT the medical history should include not only questions about alcohol intake but also explore whether recent excessive food intake has occurred.

Liver disease is often identified by asymptomatic elevations in serum aminotransferases, as these are commonly included in serum chemistry panels drawn on healthy individuals. However, incidentally discovered elevations of serum aminotransferases often lead to lengthy, expensive and potentially invasive diagnostic evaluations. Analysis of data from the Third National Health and Nutrition Examination Survey (NHANES III) in the United States showed a prevalence of elevated alanine aminotransferase (ALT) or aspartate aminotransferase (AST) to be as high as $7.9 \% .{ }^{1}$ This figure is considerably higher than the $1-4 \%$ prevalence rate reported in earlier studies, ${ }^{2}{ }^{3}$ and indicates that the prevalence of abnormal liver tests in the general population has increased. Although many cases of elevated aminotransferase levels can be explained by the consumption of large quantities of alcohol or the presence of hepatitis $\mathrm{C}$ virus (HCV) infection, a great number of cases typically remain unexplained. ${ }^{1}$ In both men and women, elevations of aminotransferases are strongly associated with features of the metabolic syndrome, such as abdominal adiposity, high plasma glucose levels and dyslipidaemia, ${ }^{4-7}$ Indeed, high serum levels of AST and ALT are associated with future risk of developing type 2 diabetes. $^{8} \quad$ Most cases of aminotransferase elevations in seemingly healthy subjects have hitherto generally been attributed to non-alcoholic fatty liver disease (NAFLD), ${ }^{19}$ and liver biopsy studies of patients referred for aminotransferase elevations have demonstrated liver steatosis as the most common histological finding. ${ }^{10-12}$ Interestingly, it was recently shown that subjects with NAFLD and elevated aminotransferases have a significant risk of developing end-stage liver disease and a lower chance of survival mainly because of cardiovascular disease. ${ }^{13}$ Accordingly, the widespread misconception that NAFLD is a mild disease with good prognosis has been highlighted ${ }^{14}$ and it is likely that physicians will be more concerned when facing patients with elevated aminotransferases. However, in most asymptomatic individuals with high aminotransferase levels, the elevation is intermittent and normal levels are found when testing is repeated within months. ${ }^{15} 16$

Although accumulation of triglycerides within hepatocytes in NAFLD is a reversible process, it is unlikely that steatosis is reversed rapidly without any specific interventions and thus can explain non-persistent aminotransferase elevations. Moreover, $79 \%$ of subjects with hepatic steatosis have normal ALT levels ${ }^{17}$ suggesting that conditions other than fatty infiltration of the liver underlie many cases of the asymptomatic elevations of aminotransferases that are commonly found in the general population.

We performed a study of the effects of 4 weeks of fast-food-based hyper-alimentation on levels of serum ALT and on hepatic triglyceride content (HTGC) measured by proton nuclear magnetic resonance spectroscopy ( $\left.{ }^{1} \mathrm{H}-\mathrm{MRS}\right)$. The aim of the study was to investigate the potential link between changes of serum ALT to the amount of hepatic fatty infiltration in healthy non-obese subjects 
during a positive energy balance resulting in a weight gain of 5$15 \%$.

\section{METHODS \\ Intervention group}

By local advertising we recruited 12 men and six women as volunteers for the intervention arm of the study. All subjects except one were students, the majority of whom were medical students. All participants had to be willing to accept an increase in body weight of $5-15 \%$ and to eat at least two fast-food-based meals a day, preferably at well known fast-food restaurants. The cost for the food was consecutively reimbursed based on the food receipts. Physical activity was not to exceed 5000 steps per day. If a study subject reached a weight gain of $15 \%$ he or she terminated the study as soon as possible by re-performing the same study investigations as were done at baseline. The participants were free from current diseases as judged by medical check-up and history. Hepatitis B surface antigen (HBsAg) and HCV antibodies were not detectable in any subject. One of the participants had many years ago been diagnosed with coeliac disease and underwent a duodenal biopsy at the end of the study. This biopsy showed normal duodenal histology.

All subjects in the intervention group continually had contact with dieticians, by weekly meetings or by phone, during the study. The dietary advice was individually adjusted to result in an intake corresponding to doubling the present caloric requirement. If the subject was not able or willing to ingest the hamburger-based diet at any stage, it was changed to whatever food the participant accepted with the highest priority to achieve the calculated caloric intake and also, if the study subject still found it acceptable, a diet rich in protein and saturated animal fat. Habitual weekly alcohol consumption was assessed at study entry and all subjects were asked to keep alcohol intake unchanged during the study period. One of the participants was an abstainer and one subject consumed $340 \mathrm{~g} /$ week. The remaining 16 subjects reported an alcohol consumption of $<140 \mathrm{~g} /$ week, which is the cut-off limit most often used to set the diagnosis of NAFLD. ${ }^{18}$

The composition of the diet was determined based on reports from 3 days before the study and another two 3-day periods at the end of the first or third weeks (or a week earlier in the one subject who ended the trial after 2 weeks). Food items that were bought in supermarkets to be consumed at less precise time points, such as butter and other foodstuffs to mainly be eaten at home, were averaged for the whole study period. In most cases the exact food composition given by the corresponding fast-food restaurant could be used as the source of information, but if such information was incomplete, food composition charts were used instead. The total caloric intake was also determined for the whole study period, and was based on receipts and interviews.

Blood for laboratory tests was drawn in the fasting state at baseline, i.e. before starting on the extra caloric intake, after 2 weeks on the fast-food-based diet, and at the end of the study, i.e. either at the end of fourth week or earlier if prematurely terminated. Blood was also drawn in the non-fasting state at the end of the first and the third study weeks, to monitor changes in serum liver enzyme levels, and at long-term followup after 6 months (in the fasting state) after the intervention, analysed as described elsewhere. ${ }^{19}$

Subjects of the intervention group underwent ${ }^{1} \mathrm{H}-\mathrm{MRS}$ of HTGC $^{20}$ by using a Philips Achieva $1.5 \mathrm{~T}$ magnetic resonance system (Philips Medical Systems, Best, The Netherlands) with a

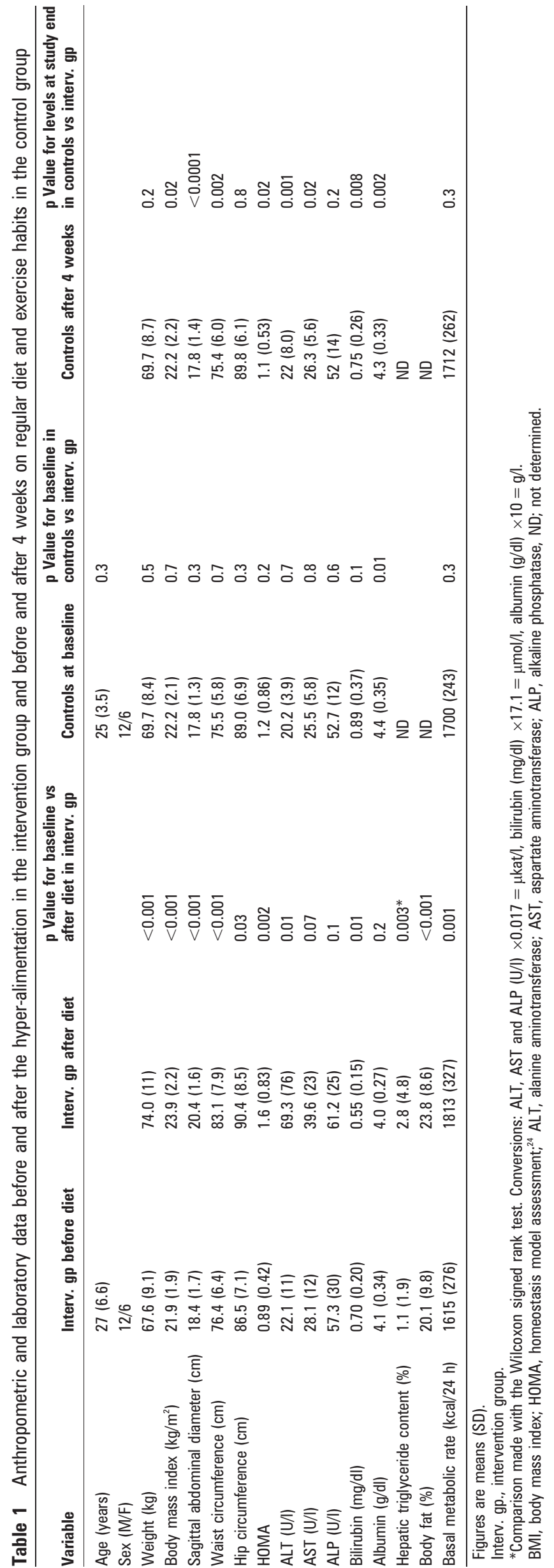


single detection element of a four element SENSE body coil. Localiser imaging for prescription of ${ }^{1} \mathrm{H}-\mathrm{MRS}$ from two different volumes of interest (VOIs) were acquired using a balanced, fast field echo pulse sequence. The ${ }^{1} \mathrm{H}-\mathrm{MRS}$ acquisition parameters were: PRESS volume selection $20 \times 20 \times 20 \mathrm{~mm}^{3}$ VOI, repetition time $=3 \mathrm{~s}$, echo time $(\mathrm{TE})=35 \mathrm{~ms}$, dummy excitations $=2$ and averages $=8$ ). All spectral and imaging acquisitions including preparation phases were performed during single breath holds. Additional spectra using a TE of $50 \mathrm{~ms}$ were acquired in a limited number of examinations for determination of a correction factor for $\mathrm{T}_{2}$ relaxation of the lipid signal. A correction factor for the $T_{2}$ relaxation time of the liver water signal was measured using an axially prescribed single slice multi-echo turbo spin echo pulse sequence, $(\mathrm{TE}=10,20,30$, 40,50 and $60 \mathrm{~ms}$ ). Zero- and first-order phase corrections, a $10 \mathrm{~Hz}$ line broadening, and a least squares optimised spline baseline correction were applied. The water and lipid resonances were integrated from 6.0 to $3.3 \mathrm{ppm}$ and 2.7 to $0.4 \mathrm{ppm}$, respectively. The lipid volume fractions (HTGC) were calculated as previously described. ${ }^{20}$ The upper limit of normal for HTGC was based on the results by Szczepaniak et al ${ }^{21}$ who examined the distribution of HTGC with ${ }^{1} \mathrm{H}-\mathrm{MRS}$ in 345 subjects with a low risk for hepatic steatosis, i.e. BMI $<25 \mathrm{~kg} /$ $\mathrm{m}^{2}$, no glucose intolerance or excessive alcohol consumption and normal serum ALT. ${ }^{22}$ Hepatic steatosis was defined as an HTGC greater than $5.6 \%$, which corresponded to the $95^{\text {th }}$ percentile in this low-risk group.

Basal metabolic rate was measured using a ventilated hood technique (Delta Trac, SensorMedics, Yorba Linda, CA, USA) ${ }^{23}$ in the fasting state in the morning and the mean value of one measurement per minute during the last $6 \mathrm{~min}$ of a $15 \mathrm{~min}$ period was calculated. The subjects were also subjected to dual energy $x$ ray absorptiometry (Hologic 4500, Hologic, Waltham, MA, USA), for analysis of body composition. A biopsy of subcutaneous abdominal fat was done under local anaesthesia and non-invasive measurements of the vascular function were recorded, the results of which will be reported separately. All the investigations were performed at baseline and during the last week of the study, except for the recording of basal metabolic rate, which was also measured in the fasting state after 2 weeks on the diet. All anthropometric measurements were done by two research nurses.

\section{Control group}

An age- and gender-matched control group was also recruited mainly to allow discrimination of changes of ALT induced by food intake from random fluctuations. The control group performed the laboratory investigations and anthropometric measurements at baseline and after 4 weeks, as well as measurement of basal metabolic rate.

\section{Statistics}

Statistical calculations were done with SPSS 14.0 software (SPSS Inc. Chicago, IL, USA). Linear correlations were calculated, except as stated in the text. Comparisons within and between groups were done with the Student paired and unpaired two-tailed t test or as stated in the results section. Mean (SD) is given unless otherwise stated. Statistical significance was considered at the $5 \%$ level $(p \leqslant 0.05)$.

\section{Ethics}

The study was approved by the Ethics Committee of Linköping University and performed in accordance with the Declaration of 
Helsinki. Written informed consent was obtained from all participating subjects.

\section{RESULTS}

Seventeen of the 18 participants met the goal of a $5-15 \%$ increase in body weight by the intervention based on hyperalimentation combined with a sedentary life style. Table 1 shows baseline anthropometric and laboratory data of all the participants, and the effects of the intervention.

The mean daily caloric intake of the whole intervention period increased $+70(35) \%($ men $+68(31) \%$, women $+74(45) \%$, $\mathrm{p}=0.8$ for comparison of genders). Two men and two women had a mean daily caloric intake $>+90 \%$ of basal during the entire study. There was no statistically significant change in the food intake of macronutrients or fibre when comparing the registration in the first and third weeks nor were there any gender differences with regard to relative intake of macronutrients. Table 2 displays food intake and the relative composition of the food before and during the study in the intervention group.

Five of the 18 subjects in the intervention group reached the maximal 15\% increase in body weight (four men and one woman). Three of these subjects reached the maximum weight increase ahead of the last study week and subsequently stopped the intervention prematurely. The subject with the steepest increase in body weight was $80 \mathrm{~kg}$ at baseline and reached $92 \mathrm{~kg}$ $(+15 \%)$ after 2 weeks. The great majority of the participants complained of epigastric discomfort and profound sense of satiety during the first week, while the remaining weeks in most cases were much more tolerable in this sense.

All subjects were informed of the test results on a weekly basis, in particular ALT levels. Figure 1 displays the ALT levels during the study in men and women. The one man who reached the highest ALT after just 1 week of hyper-alimentation (164 U/l, fig 1A) was a total abstainer from alcohol. One male participant developed an ALT level of $447 \mathrm{U} / 1$ during the third week, and thus was immediately returned to his regular eating habits. However, there were no signs or symptoms of impaired hepatic function (albumin, prothrombin time, alkaline phosphatase, AST and bilirubin were not affected) either in his case or in the other participants that displayed particularly large increases in ALT levels. Furthermore, the ALT elevations subsided either during the study (fig 1) or within a few weeks after the study had finished, in all cases. As shown in table 3 most participants developed pathological ALT levels during the study. Long-term follow-up of ALT after 6 months displayed similar levels as at baseline (baseline 22.4 (12) U/1, follow-up at 6 months 25.5 (22) $U / 1, p=0.4$ in the paired test, $n=17$, see also table 3$)$.

The increase in ALT was unrelated to the change in waist circumference but tended to correlate with the weight increase (ratio of ALT at study end/baseline ALT to the increase in weight, $r=0.42, p=0.085)$. The increase in caloric intake was not correlated with changes in ALT levels (either change from basal level to highest individual ALT, or to ALT change from basal levels to those at study end) or HTGC. The average consumption of fat or proteins during 3 days at the end of the first or third weeks was unrelated to changes in ALT. However, the maximal ALT/baseline ratio correlated with carbohydrate intake during the third week $(r=0.52, p=0.03$, corresponding figures for intake during week $1, r=0.40, p=0.1)$. The intake of sugar (mono- and disaccharides) during week 3 also correlated with the maximal ALT/baseline ALT ratio (intake during the third week, $r=0.62, p=0.006$; corresponding figures for intake at the end of the first week, $r=0.45, p=0.06$ ), but this correlation was not independent of carbohydrate consumption in a multivariate statistical model (data not shown).

HTGC increased from $1.1(1.9) \%$ to $2.8(4.8) \%$ in the intervention group ( $p=0.003$ by the Wilcoxon signed rank test, table 1). The increase of HTGC did not correlate with the increase in ALT (ratio of ALT at study end/baseline ALT to HTGC at study end/baseline, $p=0.11$, Spearman) or intake of fat, carbohydrates and protein. The increase in body weight tended to relate to the increase in HTGC $(r=0.44, p=0.07)$ while the increase in the percentage of body fat was clearly related to the increase in HTGC $(r=0.81, p<0.001)$.

The control group displayed similar anthropometric data as the intervention group but had statistically significantly higher albumin throughout the study month. There was no change in ALT levels in the control group and, as shown in table 1, the baseline ALT was similar in the intervention and the control groups while the difference at the study end between the two groups was statistically significant $(p=0.001)$.

\section{DISCUSSION}

The most important finding of this study was that during the study period 13 subjects on the high caloric diet developed pathological ALT, according to the updated definition of healthy ranges. ${ }^{22}$ In most subjects this elevation was evident within the first week. Even when the higher levels used in NHANES III ${ }^{1}$ (31 and $40 \mathrm{U} / 1$ for women and men, respectively) were considered as upper reference limits for ALT, eight and nine subjects displayed elevated ALT after 1 and 4 weeks of hyper-alimentation, respectively.

All except two subjects in the intervention group had HTGC lower than $5.6 \%$ at the end of the study period, thus not fulfilling current criteria for hepatic steatosis. ${ }^{17}{ }^{21}$ However, a significant increase of HTGC occurred indicating a net retention of lipids within hepatocytes. Thus, similar mechanisms that underlie the pathogenesis of NAFLD may have been induced in the intervention group, and at least partly explain the increased serum ALT levels. Insulin resistance, which is one of the main characteristics of the metabolic syndrome, is the most reproducible

Table 3 Number of subjects in the intervention group displaying serum ALT above reference levels during the study and at follow-up

\begin{tabular}{|c|c|c|c|c|c|c|c|}
\hline ALT reference levels & $\begin{array}{l}\text { Baseline } \\
(\text { men } n=12, \\
\text { women } n=6)\end{array}$ & $\begin{array}{l}\text { Week } 1 \\
(\text { men } n=12 \\
\text { women } n=6)\end{array}$ & $\begin{array}{l}\text { Week } 2 \\
(\text { men } n=12, \\
\text { women } n=6)\end{array}$ & $\begin{array}{l}\text { Week } 3 \\
(\text { men } n=11 \\
\text { women } n=6)\end{array}$ & $\begin{array}{l}\text { Week } 4 \\
\left(\text { men } n=11^{*}\right. \\
\text { women } n=6)\end{array}$ & $\begin{array}{l}\text { Persistent elevation } \\
\text { from week } 1 \text { to } \\
\text { study end }\end{array}$ & $\begin{array}{l}\text { Follow-up after } \\
6 \text { months } \\
\left(n=17^{* *}\right)\end{array}$ \\
\hline Men $>40 \mathrm{U} / 1 \dagger$ & 1 & 7 & 5 & 6 & 8 & 5 & 1 \\
\hline Men $>30 \mathrm{U} / \mathrm{l}+\dagger$ & 2 & 9 & 11 & 10 & 10 & 8 & 3 \\
\hline Women >31 U/l† & 0 & 2 & 3 & 3 & 2 & 1 & 0 \\
\hline Women $>19 \mathrm{U} / \mathrm{l}+\dagger$ & 1 & 5 & 4 & 3 & 4 & 3 & 2 \\
\hline
\end{tabular}

* One subject that had returned to regular eating habits during the fourth week is included.

**One subject lost to follow-up due to studies abroad.

†Upper reference limits for ALT used in NHANES III. ${ }^{1}$

†Updated upper reference limits for ALT. ${ }^{22}$ 
Figure 1 ALT levels at baseline and during the study in men $(A, n=12)$ and women $(B, n=6)$ of the intervention group.

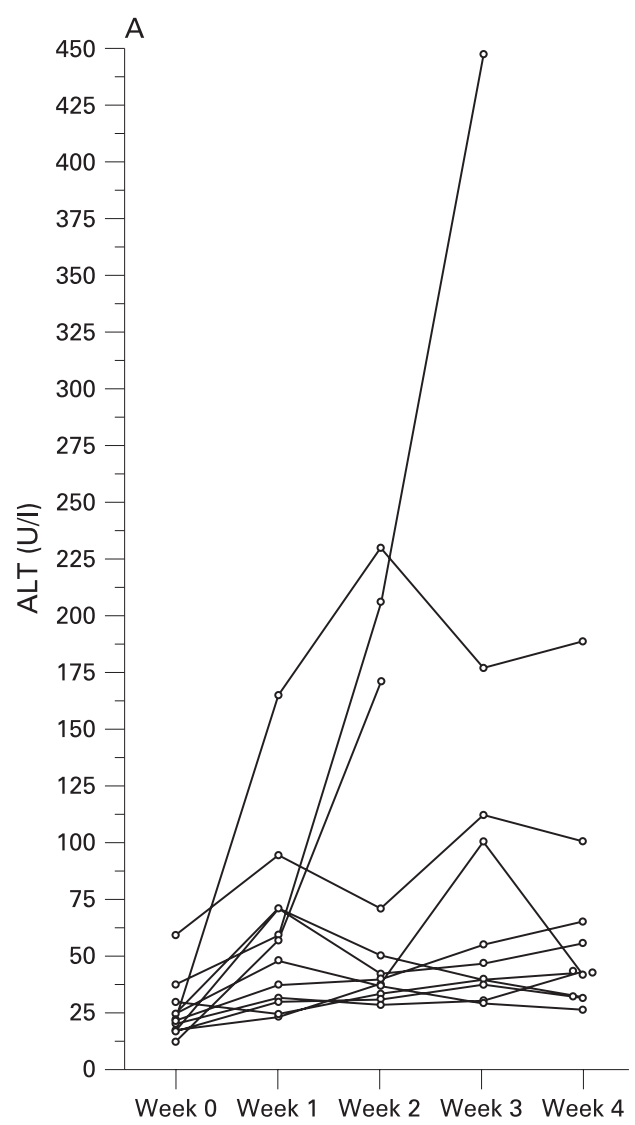

factor in the development of NAFLD. Interestingly, insulin resistance according to homeostasis model assessment (HOMA) ${ }^{24}$ increased significantly in the intervention group during the study period. Moreover, the dietary intervention also induced a visceral accumulation of adipose tissue as shown by the significant increase of waist circumference during the trial. Insulin resistance leads to the accumulation of fat in hepatocytes by two main mechanisms: lipolysis, particularly from visceral adipose tissue, which increases fatty acid concentration in the portal circulation; and hyperinsulinaemia, which leads to increased uptake of fatty acids by the hepatocytes. The increase of fatty acids within hepatocytes may have led to hepatocellular injury, depicted by increased serum ALT in the participating subjects, through multiple mechanisms (for a review see Angulo ${ }^{18}$ ). It has recently been suggested that hepatocellular injury in NAFLD may be attributable to the combined effects of severe peripheral insulin resistance and relative failure of humoral (adipokine) mediators that combat the effect of high insulin levels on hepatic lipid turnover. ${ }^{25}$ However, ALT levels increased already within a week, a time period after which a significant increase of lipids within hepatocytes seems unlikely to have developed. Furthermore, the magnitude of ALT elevation only tended to correlate with those of HTGC, making it unlikely that fatty infiltration of the liver was the main cause of the rapidly increased ALT levels that were evident in many subjects. Hepatocellular damage due to other reasons than intracellular accumulation of lipids, for example localised inflammation, could potentially explain the elevated ALT levels. Indeed, when examining the relationship of the increase in ALT to intake of different nutrients, fat intake was unrelated to increase in ALT while sugar and carbohydrate intake at week 3 clearly related to the ALT increase. This is in accordance with earlier findings by Solga et al who demonstrated that higher carbohydrate intake was significantly associated with an increased risk of biopsy-proven hepatic inflammation in morbidly obese patients undergoing bariatric surgery. ${ }^{26}$ Several participants displayed a spontaneous decrease of ALT levels after the initial increase, during the study, while still gaining weight by the intervention, which is surprising if inflammation was the major mediator of the ALT increase. We believe that a plausible explanation for the ALT elevations in this study may be that the sudden increased supply of metabolic substrates, to the liver, in particular monosaccharides, caused an enzymatic induction in hepatocytes and hence that the normal leakage of ALT through the cell membrane resulted in elevated serum levels. The fact that bilirubin levels decreased in the intervention group is in line with induction of hepatic metabolic capacity rather than frank liver cell damage.

Close clinical follow-up has been suggested as the most costeffective strategy for asymptomatic patients with negative tests for viral, metabolic and autoimmune markers of liver disease and chronically elevated aminotransferase levels. If the levels are spontaneously normalised or only intermittently elevated, no further evaluation is usually undertaken. However, in many cases high alcohol consumption is suspected even though shortterm ingestion of alcohol in healthy individuals has not been shown to be associated with clinical significant increases of aminotransferases. ${ }^{27}{ }^{28}$ Interestingly, in a study by Belfrage et al daily ingestion of $63 \mathrm{~g}$ alcohol for 5 weeks induced fatty infiltration of the liver but not elevations of aminotransferases. ${ }^{29}$ In comparison, our study showed that 4 weeks of hyperalimentation is associated with clinically significant elevations of aminotransferases in many subjects but with development of hepatic steatosis in just one subject out of 18 .

The major weakness of our study is that subjects did not undergo liver biopsy to confirm the absence of hepatic pathology at baseline. However, 15 of 18 subjects had normal 
baseline ALT levels according to the updated definitions ${ }^{22}$ and all subjects except one had HTGC well below the level considered to be abnormal, i.e. $5.6 \%,{ }^{21}$ and none had antibodies indicative of viral hepatitis. Thus, underlying liver disease is unlikely among the participating subjects. One subject with an HTGC of $8.7 \%$ reported an average weekly alcohol consumption of $340 \mathrm{~g}$, and thus he was considered to have alcoholic steatosis. However, his baseline ALT was also normal (21 U/l) according to the updated definitions. Due to the demanding and unusual nature of the trial it was not possible to recruit enough potential intervention participants to allow randomisation to the intervention or control groups. It should also be noted that the participants were not representative of the general Swedish citizen, as displayed, for example, by the high baseline fibre intake, and of course by inclusion of only lean subjects. Although the levels of serum aminotransferases were the same in controls and in the subjects of the intervention group at baseline, we were not able to analyse this in relation to the diet composition in the controls, since this was not recorded.

Automated routine laboratory testing is frequently part of an annual check-up and physicians are often faced with the problem of a patient with abnormal result on measurement of serum ALT but no symptoms. We conclude that chronically or intermittently elevated ALT can be of purely nutritional origin, particularly when found in the absence of liver steatosis. The fact that 14 out of 18 participants had pathological ALT levels after just 1 week clearly displays that elevated ALT levels after a short over-indulgent holiday can be caused not only by alcohol ingestion, but also by a higher caloric intake than usual combined with a sedentary behaviour. We suggest that in the clinical evaluation of subjects with elevated ALT physicians should include not only questions about alcohol intake, but also explore whether recent excessive food intake has occurred.

Acknowledgements: In addition to the main authors of the manuscript the Fast Food Study Group consisted of Dr Preben Kjölhede, Division of Obstetrics and Gynaecology, Dr Anneli Sepa, Division of Pediatrics, both at the Department of Molecular and Clinical Medicine; Professor Peter Strålfors Department of Cell Biology, Dr Gunilla Hollman and Professor Toste Länne, Department of Medicine and Care, all five being at the Faculty of Health Science, Linkoping University; and Dr Martin Carlsson, Department of Internal Medicine, County Hospital of Kalmar.

Funding: The study was supported by University Hospital of Linkoping Research Funds, Linkoping University, Gamla Tjänarinnor, Medical Research Council of Southeast Sweden and the Diabetes Research Centre of Linkoping University. The funding sources had no impact on the design or performance of the study.

Competing interests: None.

\section{REFERENCES}

1. Clark JM, Brancati FL, Diehl AM. The prevalence and etiology of elevated aminotransferase levels in the United States. Am J Gastroenterol 2003;98:960-7.

2. Bailey A. Biochemistry of well populations. Lancet 1974;2:1436-9.

3. Bates B, Yellin JA. The yield of multiphasic screening. JAMA 1972;222:74-8.

4. Diehl AM. Nonalcoholic steatohepatitis. Semin Liver Dis 1999;19:221-9.
5. Powell EE, Cooksley WG, Hanson R, et al. The natural history of nonalcoholic steatohepatitis: a follow-up study of forty-two patients for up to 21 years. Hepatology 1990;11:74-80.

6. Bacon BR, Farahvash MJ, Janney CG, et al. Nonalcoholic steatohepatitis: an expanded clinical entity. Gastroenterology 1994;107:1103-9.

7. Marchesini G, Brizi M, Morselli-Labate AM, et al. Association of nonalcoholic fatty liver disease with insulin resistance. Am J Med 1999;107:450-5.

8. Hanley AJ, Williams K, Festa A, et al. Elevations in markers of liver injury and risk of type 2 diabetes: the insulin resistance atherosclerosis study. Diabetes 2004; 53:2623-32.

9. Yu AS, Keeffe EB. Elevated AST or ALT to nonalcoholic fatty liver disease: accurate predictor of disease prevalence? Am J Gastroenterol 2003:98:955-6.

10. Mathiesen UL, Franzen LE, Fryden A, et al. The clinical significance of slightly to moderately increased liver transaminase values in asymptomatic patients. Scand J Gastroenterol 1999:34:85-91.

11. Skelly MM, James PD, Ryder SD. Findings on liver biopsy to investigate abnormal liver function tests in the absence of diagnostic serology. J Hepatol 2001;35:195-9.

12. Daniel S, Ben-Menachem T, Vasudevan G, et al. Prospective evaluation of unexplained chronic liver transaminase abnormalities in asymptomatic and symptomatic patients. Am J Gastroenterol 1999:94:3010-4.

13. Ekstedt M, Franzen LE, Mathiesen UL, et al. Long-term follow-up of patients with NAFLD and elevated liver enzymes. Hepatology 2006;44:865-73.

14. Ratziu V, Poynard T. Assessing the outcome of nonalcoholic steatohepatitis? It's time to get serious. Hepatology 2006;44:802-5.

15. Friedman LS, Dienstag JL, Watkins $\mathrm{E}$, et al. Evaluation of blood donors with elevated serum alanine aminotransferase levels. Ann Intern Med 1987;107:137-44.

16. Kundrotas LW, Clement DJ. Serum alanine aminotransferase (ALT) elevation in asymptomatic US Air Force basic trainee blood donors. Dig Dis Sci 1993;38:214550.

17. Browning JD, Szczepaniak LS, Dobbins R, et al. Prevalence of hepatic steatosis in an urban population in the United States: impact of ethnicity. Hepatology 2004;40:1387-95.

18. Angulo P. Nonalcoholic fatty liver disease. N Engl J Med 2002;346:1221-31.

19. Stromme JH, Rustad P, Steensland H, et al. Reference intervals for eight enzymes in blood of adult females and males measured in accordance with the International Federation of Clinical Chemistry reference system at 37 degrees C: part of the Nordic Reference Interval Project. Scand J Clin Lab Invest 2004;64:371-84.

20. Longo $\mathbf{R}$, Pollesello $\mathrm{P}$, Ricci $\mathrm{C}$, et al. Proton MR spectroscopy in quantitative in vivo determination of fat content in human liver steatosis. J Magn Reson Imaging 1995; 5:281-5.

21. Szczepaniak LS, Nurenberg P, Leonard D, et al. Magnetic resonance spectroscopy to measure hepatic triglyceride content: prevalence of hepatic steatosis in the general population. Am J Physiol Endocrinol Metab 2005;288:E462-8.

22. Prati D, Taioli E, Zanella $A$, et al. Updated definitions of healthy ranges for serum alanine aminotransferase levels. Ann Intern Med 2002;137:1-10.

23. Lof $\mathbf{M}$, Olausson $\mathrm{H}$, Bostrom $\mathrm{K}$, et al. Changes in basal metabolic rate during pregnancy in relation to changes in body weight and composition, cardiac output, insulin-like growth factor I, and thyroid hormones and in relation to fetal growth. Am J Clin Nutr 2005:81:678-85.

24. Bonora E, Targher $\mathrm{G}$, Alberiche $\mathrm{M}$, et al. Homeostasis model assessment closely mirrors the glucose clamp technique in the assessment of insulin sensitivity: studie in subjects with various degrees of glucose tolerance and insulin sensitivity. Diabetes Care 2000;23:57-63.

25. Larter CZ, Farrell GC. Insulin resistance, adiponectin, cytokines in NASH: Which is the best target to treat? J Hepatol 2006;44:253-61.

26. Solga $\mathbf{S}$, Alkhuraishe AR, Clark JM, et al. Dietary composition and nonalcoholic fatty liver disease. Dig Dis Sci 2004:49:1578-83.

27. Rubin E, Lieber CS. Alcohol-induced hepatic injury in nonalcoholic volunteers. N Engl J Med 1968;278:869-76.

28. Brohult J, Carlson LA, Reichard H. Serum-enzyme activities, cholesterin, and triglycerides in serum after intake of alcohol. Scand J Clin Lab Invest Supp/ 1966:18:82-3

29. Belfrage $\mathbf{P}$, Berg B, Cronholm T, et al. Prolonged administration of ethanol to young healthy volunteers: effects on biochemical, morphological and neurophysiological parameters. Acta Med Scand Supp/ 1973:552:1-44. 